\title{
Culture in vitro d'embryons isolés de noyer commun (Juglans regia L.)
}

\author{
C. JAY-ALLEMAND et D. CORNU \\ INRA, Station d'Amélioration des Arbres forestiers \\ Ardon, $F 45160$ Olivet
}

\begin{abstract}
Résumé
La technique in vitro de production de pousses à partir des axes embryonnaires de noyer dépend successivement de deux facteurs :

- la concentration en saccharose qui, utilisée à 20 ou $40 \mathrm{~g} / 1$, permet d'obtenir des plantules enracinćes sur un milicu simple de KNOP dilué de moitié, après 60 jours de culture ;

- la $\mathrm{N}_{\mathrm{i}}$-benzylaminopurine à $1 \mathrm{mg} / \mathrm{l}$ qui déclenche après 30 jours de culture la formation de pousses axillaires sur des épicotyles excisés des plantules précédentes el ensemencés sur le milieu de MıLıer à $20 \mathrm{~g} / 1$ de saccharose.
\end{abstract}

Mots clés : Cultures in vitro dembryon, cytokinine, sucre, Juglans regia.

\section{Introduction}

Dans le cadre d'un programme d'amélioration du noyer à bois, unc valorisation accélérée des embryons hybrides créés par fécondation contrôlće présente un grand intérêt. Ainsi, l'obtention de jeunes pousses par la technique de culture in vitro d'embryons isolés doit permettre, tout en réduisant les pertes observées lors de la germination des noix hybrides, d'aboutir rapidement à une multiplication végétative in vitro efficace.

Plusieurs travaux ont été effectués sur d'autres espèces dans ce sens : HanNig (1904), Tuckey (1933-1934), Mclean (1946), Stone \& Duffiel.d (1950). Lammerst en 1942 utilisait déjà cette méthode pour raccourcir le cycle de reproduction sexuće des jeunes arbres.

La composition en sels minéraux des milieux de culture (Bouharmont, 1961, Monnier, 1973-1976), les apports de vitamines et surtout de saccharose (LF. PageDegivry, 1967, sur Ginkgo biloba et Taxus baccata, Thomas, 1970-1980 sur Pinus sylvestris), semblent indispensables pour obtenir un développement complet des embryons immatures ou matures cultivés in vitro. 
L'acide gibbérellique (GA) est fréquemment utilisé soit en tant qu'activateur ou inducteur enzymatique (SIMPSON, 1965), soit directement en tant que facteur de levée de dormance (Bulard \& Monin, 1960 a-b, Raghavan \& Torrey, 1964, Bouvinet \& Rabechault, 1965, Le Page-Degivry, 1973 a-b). Plus récemment, la $\mathrm{N}$ Benzylaminopurine (BAP) incorporée dans le milieu de culture d'embryons matures de châtaignier a permis le développement de nombreuses pousses axillaires (VIEITEZ \& Vieitez, 1980 b).

\section{Matériel et méthodes}

\subsection{Matériel végétal}

Des noix de Juglans regia libérées de leur brou et séchées naturellement, ont été ramassées au mois d'octobre 1981 et conservées à la température du laboratoire. Inséré entre les deux cotylédons, l'embryon a une longueur de 3 à $4 \mathrm{~mm}$ et sa largeur est proche de $2 \mathrm{~mm}$.

L'excision des embryons s'effectue dans des conditions stériles. Les cerneaux sont désinfectés dans une solution d'hypochlorite de Calcium à $60 \mathrm{~g} / 1$ pendant $20 \mathrm{mn}$ et rincés 3 fois à l'eau stérile. Prolonger le dernier rinçage pendant 3 heures augmente l'imbibition des cotylédons et facilite le prélèvement des embryons.

\subsection{Conditions de culture}

Les milieux de culture, autoclavés à $116^{\circ} \mathrm{C}$ pendant $30 \mathrm{mn}$, sont constitués par les macroćléments de KNOP dilués de moitié ou de MiLrer (1967), les microéléments de Murashige \& Skoog (1962) ou de Miller (1967), la biotine $(0,01 \mathrm{mg} / 1)$, la glutamine $(200 \mathrm{mg} / \mathrm{l})$, le myo-inositol $(100 \mathrm{mg} / \mathrm{l})$ et de $0,1 \mathrm{mg} / 1 \mathrm{~d}$ 'acide nicotinique, de pyridoxine, de thiamine, de pantothenate de Calcium, de L-Cystéine. Les milieux sont solidifiés à l'aide du Difco-bacto agar à $7 \mathrm{~g} / 1$ et le $\mathrm{pH}$ ajusté à 5,6 . Les concentrations de saccharose et de régulateurs de croissance $\mathrm{N}_{6}$ Benzylaminopurine (BAP) et acide gibbérellique $\left(G_{*}\right)$ sont modifiées selon l'expérimentation. Les embryons ensemencés en tube $(20 \mathrm{ml}$ de milieu par tube) sont placés en chambre de culture à $25^{\circ} \mathrm{C}$ tout d'abord à l'obscurité (Diamantoglou \& Mitrakos, 1979) puis en photopériode de 16 heures sous un éclairement de $33 \mathrm{w} / \mathrm{m}^{2}$ environ.

\subsection{Critères de développement et de croissance}

Pour chaque embryon ensemencé, une fois par semaine est mesuré l'allongement des parties aériennes et racinaires et au bout de 50 jours de culture est compté le nombre de feuilles et de racines secondaires apparues. Après transfert sur des milieux neufs, les embryons qui présentent au bout de 30 jours des bourgeons débourrés et allongés sont dénombrés pour comparer les effets des régulateurs de croissance.

Une analyse de variance sur la croissance a permis de dégager les différences significatives au seuil de 5 p. 100. 


\section{Résultats}

\subsection{Influence de la concentration en saccharose}

72 embryons sont ensemencés simultanément sur le milieu de KNOP is et répartis uniformément en fonction de 6 concentrations différentes en saccharose $(0,10,20,40,80$ et $160 \mathrm{~g} / \mathrm{l})$.

\subsection{Elongation}

Les courbes de croissance moyenne (fig. 1) expriment l'évolution des développements aériens et racinaires :

- les faibles concentrations $(10,20,40 \mathrm{~g} / \mathrm{l})$ en sucre favorisent lallongement des épicotyles;

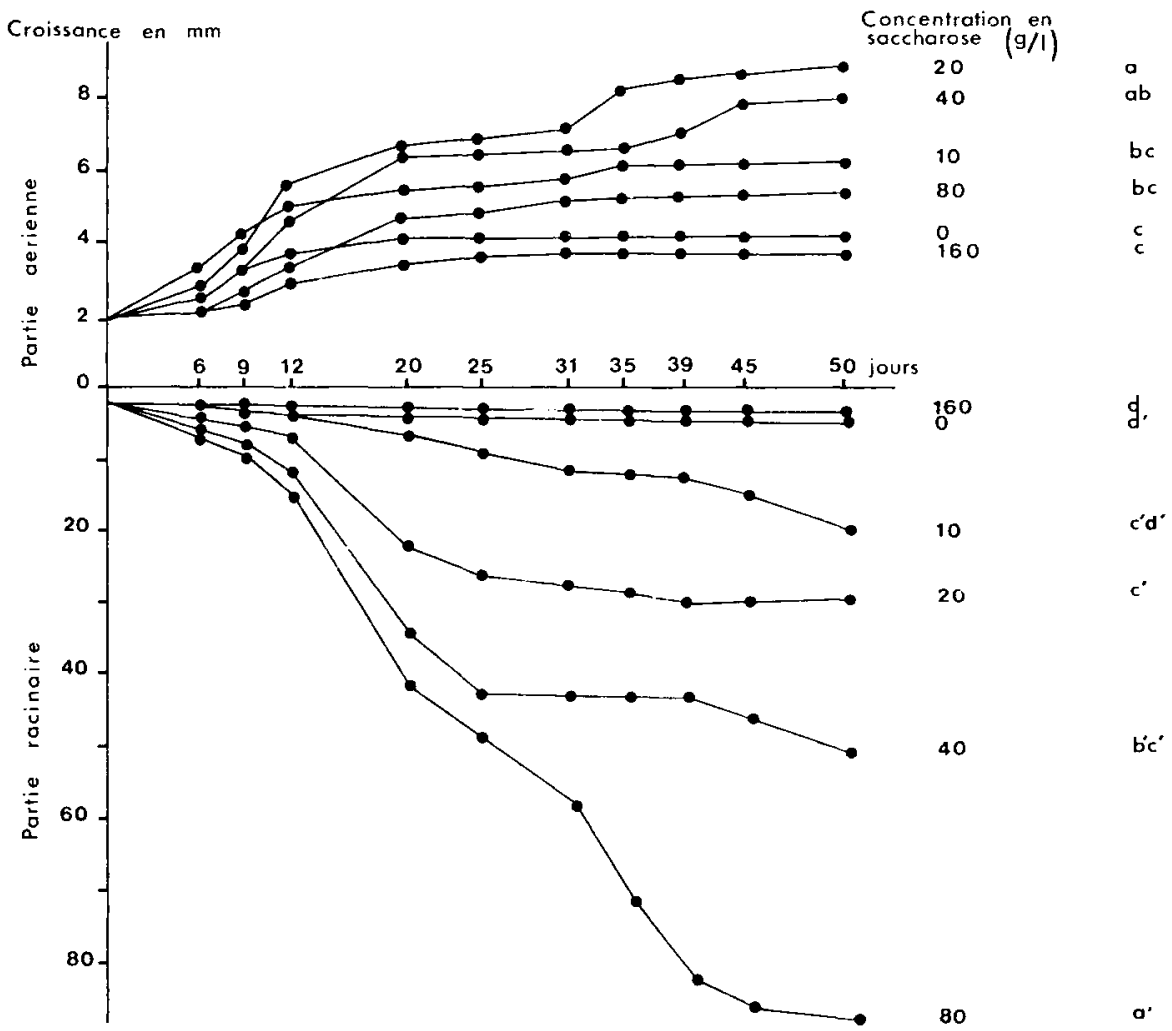

Fig. 1

Evolution de la croissance moyenne d'épicotyles et de racines principales d'embryons en fonction de la concentration des milieux en saccharose (12 embryons par traitement).

Les valeurs suivies d'une même lettre

ne sont pas significativement différentes au seuil de 5 p. 100.

Effect of sucrose concentration on epicotyl and primary root growth of isolated embryos. $(n=12$, mean lengths after 50 days followed by same symbol are not different at 5 p. 100 level). 
- à $80 \mathrm{~g} / \mathrm{l}$ de saccharose la croissance racinaire est exaltée :

- les concentrations extrêmes ( 0 et $160 \mathrm{~g} / 1)$ bloquent systématiquement le développement des embryons.

\subsection{Organogenèse foliaire et racinaire}

Elle est appréciée par le nombre de feuilles développées sur l'épicotyle et le nombre de racines secondaires (tabl. 1):

- 10,20 et $40 \mathrm{~g} / \mathrm{I}$ de saccharose favorisent la formation de feuilles;

- à $80 \mathrm{~g} / 1$ on a une augmentation du nombre de racines secondaires. Parallèlement le volume racinaire est lui aussi augmenté ;

- si aucun développement n'est noté pour les concentrations de 0 et $160 \mathrm{~g} / \mathrm{l}$, il existe cependant, dans les tous premiers jours qui suivent l'ensemencement, une réaction comparable aux autres séries : verdissement de l'épicotyle, gonflement et apparition de la jeune racine, preuve d’une certaine autonomie des embryons isolés.

\section{TABLeaU 1}

Nombre moyen de feuilles et de racines secondaires produites in vitro e'n fonction de la concentration en sacharose (exprimée en g/l) après 50 jours de culture.

Effect of sacrose concentration on production of leaves and secondary roots. (Mean number per embryo after 50 days of culture, 12 embryos per treatment).

\begin{tabular}{|c|c|c|c|c|c|c|}
\hline Concentration en saccharose en $\mathrm{g} / \mathrm{I}$ & 0 & 10 & 20 & 40 & 80 & 160 \\
\hline Nombre moyen de feuilles par embryon & 0 & 1,37 & 1,58 & 1,32 & 0,57 & 0 \\
\hline $\begin{array}{c}\text { Nombre moyen de racines secondaires par } \\
\text { embryon } \ldots \ldots \ldots \ldots \ldots \ldots \ldots \ldots\end{array}$ & 0 & 0 & 0 & 0,65 & 1,22 & 0 \\
\hline
\end{tabular}

Dans aucun cas, la formation d’une tige à partir du bourgeon apical n'est observée. Seules, deux rangées de 5 à 8 bourgeons placés symétriquement sur le dôme épicotylaire se sont nettement individualisćes. L'incorporation de BAP associée ou non avec l’acide gibbéréllique n’a pas permis d’améliorer ces développements (JAYAillemand, 1982).

\subsection{Influence des régulateurs de croissance}

A l'arde des plantules âgées de 50 jours, obtenues sur le milieu favorable au développement des parties aériennes (KNOP $\frac{1}{2}$, saccharose à $20 \mathrm{~g} / \mathrm{l}$ ), nous avons comparé sur un milieu minéral plus concentré en sels minéraux utilisé pour la culture dorganes (milieu de Milı.r. 1967), l"influence des régulateurs de croissance (BAP à $1 \mathrm{mg} / \mathrm{l}$ et $\mathrm{BAP} 1 \mathrm{mg} / \mathrm{l}+\mathrm{GA}_{33} 10 \mathrm{mg} / \mathrm{l}$ ) sur le développement de plantules entières ou privées de leurs systèmes racinaires (épicotyles excisés). Chaque traitement comporte 10 à 14 explants. Les résultats sont présentés dans le tableau 2, après 30 jours de culture sur le nouveau milieu. 


\section{TABLEAU 2}

Débourrement et allongement des bourgeons de plantules entières ou d'épicotyles excisés, après 30 jours de culture, en fonction de deux traitements en régulateur de croissance : BAP $1 \mathrm{mg} / \mathrm{l}$ et BAP $1 \mathrm{mg} / \mathrm{l}+G A, 10 \mathrm{mg} / \mathrm{l}$. (Résultats nuls en l'absence de régulateurs).

Bud development and elongation for whole embryos and excised epicotyls following two hormonal treatments : BAP $1 \mathrm{mg} / \mathrm{l} ; B A P 1 \mathrm{mg} / \mathrm{l}+\mathrm{GA}, 10 \mathrm{mg} / \mathrm{l}$ (after 30 days of culture).

Without hormone treatment there was no bud development or elongation.

\begin{tabular}{|c|c|c|c|c|}
\hline & \multicolumn{2}{|c|}{ Plantules entières } & \multicolumn{2}{|c|}{ Epicotyles excisés } \\
\hline & $\mathrm{BAP}$ & $\mathrm{BAP}+\mathrm{GA}_{3}$ & BAP & $\mathrm{BAP}+\mathrm{GA}_{\mathrm{s}}$ \\
\hline Nombre d'explants ensemencés. & 14 & 14 & 13 & 11 \\
\hline $\begin{array}{c}\text { \% d'explants ayant au moins } 1 \text { bourgeon } \\
\text { débourré } \ldots \ldots \ldots \ldots \ldots \ldots \ldots \ldots \ldots\end{array}$ & 100 & 100 & 100 & 100 \\
\hline $\begin{array}{c}\text { Nombre moyen de bourgeons débourrés } \\
\text { par explant } \ldots \ldots \ldots \ldots \ldots \ldots \ldots \ldots\end{array}$ & 4,5 & 5,2 & 7,8 & 8 \\
\hline $\begin{array}{c}\% \text { d'explant ayant au moins } 1 \text { bourgeon } \\
\text { allongé } \ldots \ldots \ldots \ldots \ldots \ldots \ldots \ldots \ldots \ldots\end{array}$ & 78 & 50 & 69 & 0 \\
\hline $\begin{array}{c}\text { Nombre moyen de bourgeons allongés par } \\
\quad \text { explant } \ldots \ldots \ldots \ldots \ldots \ldots \ldots \ldots \ldots\end{array}$ & 0,80 & 0,57 & 1,4 & 0 \\
\hline
\end{tabular}

En l’absence de régulateur de croissance, aucune réponse n`a été observéce.

Dans tous les autres cas, les explants présentent au moins un bourgeon débourré. En ce qui concerne le nombre moyen de bourgeons débourrés par traitement, il apparaît qu'indépendamment du type de traitement hormonal, la nature de l'explant semble jouer un rôle déterminant. Les épicotyles excisés présentent de meilleurs résultats que ceux des plantules entières, mettant ainsi en évidence un rôle inhibiteur des racines.

La formation de jeunes pousses, sur l'épicotyle, est favorisée par $1 \mathrm{mg} / \mathrm{l}$ de BAP. En l'absence de racines, le nombre de bourgeons allongés par explant est 2 fois plus élevé que pour les plantules entières, néanmoins, celles-ci sont plus nombreuses à posséder au moins un bourgeon allongé.

Contrairement aux résultats fréquemment observés en culture in vitro de tige, ladjonction de $10 \mathrm{mg} / \mathrm{I}$ de $\mathrm{GA}_{33}$ (avant autoclavage) au milieu de culture précédent bloque systématiquement tout développement des bourgeons présents sur les épicotyles excisés. Cette baissc de réactivité est observée dans une moindre mesure sur plantules entières. 


\section{Discussion}

\subsection{Rôle du saccharose}

Employé à différentes concentrations le saccharose oriente significativement le développement des embryons matures. Celui-ci peut dépendre à la fois d'un facteur trophique essentiel et, comme le suggèrent Rietsema, Satina \& Blakeslee (1953), de phénomènes purement physiques liés à des variations de pression osmotique au niveau racinaire.

Pour Thomas (1980), le saccharose, dans les conditions de culture in vitro, intervient sur les équilibres de croissance et sur la localisation des mitoses. Cet auteur précise qu'une concentration de $100 \mathrm{~g} / \mathrm{l}$ de saccharose ralentit les divisions cellulaires des embryons différenciés de Pinus sylvestris. D'après Ruven (1952), les fortes doses en sucre inhiberaient l'élongation cellulaire mais permettraient la poursuite de la division cellulaire et de la différenciation.

Nos résultats s'accordent avec ces différentes observations. On peut y distinguer deux phases :

- l'une où la concentration croissante de saccharose agit positivement sur le développement de l'embryon, en favorisant le métabolisme de la partie aérienne,

- l'autre où les fortes concentrations établissant de hautes pressions osmotiques, peuvent réduire (à $80 \mathrm{~g} / \mathrm{l}$ ) le transport de l'eau et des métabolites (saccharose, éléments minéraux, ...) de la racine vers la partie aérienne. Au-delà de cette dose, la croissance racinaire décroît (à $100 \mathrm{~g} / 1$, JAY-ALLEMAND, 1982) et tend à s'arrêter à $160 \mathrm{~g} / \mathrm{l}$, conséquence probable d'un blocage progressif de l'absorption racinaire.

Bien que le système racinaire présente une forte croissance, cette source carbonée, associće aux autres éléments du milieu de culture, n’assure cependant pas la formation de tiges à partir de l'épicotyle. Un séjour au froid à 4 " $\mathrm{C}$ des noix ou des embryons isolés n'a pas permis non plus l'apparition de pousses. Une accélération de la vitesse de croissance, une augmentation du nombre de feuilles et de racines secondaires ont pu être néanmoins observées.

\subsection{Rôle de la $B A P$}

L'analyse des résultats met en valeur lintérêt de la technique de culture des épicotyles excisés des plantules obtenues in vitro à partir d'embryons matures. Remarquons que Vieitez et Vieitez, $1980(\mathrm{a}, \mathrm{b})$ obtiennent directement 62 p. 100 d'axes embryonnaires de châtaigniers producteurs de pousses axillaires avec $1 \mathrm{mg} / \mathrm{l}$ de BAP après deux mois de culture, sans ablation de racines. Dans ces conditions les embryons de noyer commun ne présentent aucun allongement aérien. Une subculture d'un mois, d'épicotyles excisés d'embryons développés sur milieu KNOP $\frac{1}{2}$ saccharosé $(20 \mathrm{~g} / 1)$ suffit pour déclencher dans près de 70 p. 100 des cas l'élongation de deux bourgeons en moyenne par épicotyle.

Ces résultats montrent que la racine s'oppose à l'action de la BAP au niveau des bourgeons. Différents mécanismes peuvent être impliqués : l'absorption ou le transport d'ćléments minéraux ou de régulateur de croissance n'ont pas lieu, la racine 
peut être productrice parallèlement d'inhibiteurs de croissance, de type hormonal ou phénolique par exemple... Finalement, on peut se demander si les racines, sièges de la synthèse de cytokinines, sont réellement fonctionnelles lorsqu'elles sont formées in vitro.

Seule une éude plus fine de caractère biochimique, permettrait de choisir entre ces différentes hypothèses.

Dans cette étude nous avons défini les conditions favorables à la production de pousses in vitro à partir d'embryons de noyer. Deux étapes sont nécessaires :

1) obtention de plantules à partir d'axes embryonnaires ensemencés sur milieu de KNOP dilué de moitié avec 20 ou $40 \mathrm{~g} / 1$ de saccharose durant 50 jours;

2) culture des épicotyles excisés des plantules précédentes âgées de 2 mois durant 30 jours sur milieu de Miller à $20 \mathrm{~g} / \mathrm{I}$ de saccharose additionné de $1 \mathrm{mg} / \mathrm{l}$ de BAP.

Cette technique peut permettre ainsi de sauvegarder, en conditions aseptiques et sous forme de jeunes pousses, des plants hybrides potentiellement performants. C'est aussi, par la culture des épicotyles excisés, la première étape indispensable pour la mise en place d'un processus de multiplication in vitro accélérée et à grande échelle de ces hybrides.

\author{
Reçu en mai 1985. \\ Accepté en octobre 1985.
}

\begin{abstract}
Summary
In vitro culture of isolated embryos of walnut (Juglans regia L.)
\end{abstract}

The main objective of the walnut improvement program of the Forest Tree Improvement Station (INRA-Orléans) is the vegetative propagation of hybrids created by controlled cross. For that purpose, establishment of an in vitro micropropagation technique from embryos could save much time.

In this work, we present the technique used to obtain good development of isolated embryos of Juglans regia L. Two main factors studied were the concentration of sucrose in the culture medium and the effect of $\mathrm{N}_{t}$-Benzylaminopurine.

\title{
1) Sucrose effect
}

Naked embryos were transferred from disinfected nuts to Knop's medium supplemented with sucrose at $0,10,20,40,80$ and $160 \mathrm{~g} / 1$. After 50 days of culture (tabl. 1 and fig. 1) the best growth of epicotyls and roots was obtained with $20 \mathrm{~g} / 1$ of sucrose. Low concentration (less than $40 \mathrm{~g} / \mathrm{l})$ promoted epicotyl growth whereas high concentration $(80 \mathrm{~g} / 1)$ increased root growth. When sucrose was absent or at too high concentration (160 g/l) embryo development did not occure.

In all treatments the epicotyls of embryos formed a terminal bud and 10 to 16 lateral buds in two rows. None of those buds flushed.

\section{2) Effect of $N_{6}$-Benzylaminopurine}

The developed embryos obtained on the medium showing the best growth (knop $1 / 2$ and sucrose $20 \mathrm{~g} / \mathrm{l}$ ) were transferred, with and without roots to the medium of Miller supplemented or not with BAP $1 \mathrm{mg} / 1$ or BAP $1 \mathrm{mg} / 1$ and $\mathrm{GA}_{3} 10 \mathrm{mg} / 1$. Flushing of at least one bud per plant occured only if there is BAP in the medium (tabl. 2). On BAP alone the mean number of buds elongated per explant was about 2 times greater on excised epicotyls than on whole embryos. There was a depressive effect of $\mathbf{G A}_{\text {: }}$ on 
elongation of buds particularly on excised epicotyls. Apparently there is a strong effect of roots on the development of buds.

With this technique, we are able to establish directly walnut embryos in in vitro culture, the first step for a mass micropropagation process.

Key words : In vitro embryo culture, cytokinin, sucrose, Juglans regia.

\section{Références bibliographiques}

Bouharmont J., 1961. Embryo culture of rice on sterile medium. Euphytica, 10, 283-293.

Bouvinet J., Rabechault M., 1965. Effets de l'acide gibbérellique sur les embryons du palmier à huile en culture «in vitro». C.R. Acad. Sci. Paris, 260, 5336-5338.

Bulard C., Monin J., 1960 a. Action de l'acide gibbérellique sur des embryons dormants d'Evonymus europaeus cultivés « in vitro». C.R. Acad. Sci. Paris, 250, 2922-2924.

Bulard C., Monin J., 1960 b. Graines et embryons dormants d'Evonymus europaeus différentes modalités dans l'éveil de leur dormance par l'acide gibbérellique, C.R. Acad. Sci. Paris, 250, 4107-4199.

Diamantoglou S., Mitrakos K., 1979. Sur la culture « in vitro » de l'embryon d'olivier (Olea europea L. var. Oleaster). C.R. Acad. Sci. Paris, 288, 1537-1540.

HanNig E., 1904. Zur Physiologie pflanzlicher Embryonen. I. Über die Kultur von Cruciferen Embryonen ausserhals des Embryosachs. Bot. Ztg., 62, 45-80.

Jay-Allemand C., 1982. Culture in vitro du Noyer (Juglans sp.). Etude expérimentale sur l'ensemencement d'embryons isolés et de bourgeons. D.E.A. Agronomie U.S.T.L. de Montpellier, $125 \mathrm{p}$.

LAMmERST W.E., 1942. Embryo culture, an effective technique for shortening the breeding cycle of deciduous trees and increasing germination of hybrid seed. Am. J. Bot., 29, $166-171$.

LePage-Degivry M.T., 1967. Développement «in vitro» d'embryons encore immatures an moment de la dissémination des semences chez quelques plantes ligneuses. Thèse de 3* Cycle, Paris, 122 p.

Lepage-Degivry M.T., 1973 a. Influence de l'acide abscissique sur le développement des embryons de Taxus baccata L. cultivés «in vitro». Zeit. Pflanz, 70, 406-413.

LePAGE-Degivry M.T., 1973 b. Intervention d'un inhibiteur lié dans la dormance embryonnaire de Taxus baccata. C.R. Acad. Sci. Paris, 277, 177-180.

MCLEAN S.W., 1946. Interspecific crosses involving Datura ceratocaula obtained by embryo dissection. Am. J. Bot., 33, 630-638.

Miller C.O., 1967. Cytokinin in Zea mays. Annals of the New York. Acad. Sci., 144 (1), 250-257.

MonNitik M., 1973. Croissance et développement des embryons globulaires de Capsella bursa pastoris cultivés «in vitro» dans un milieu à base d'une nouvelle solution minérale. Soc. Bor. Fr. Mémoires 1973, Coll. Morphologie, 179-194.

Monnier M., 1976. - Variations des besoins nutritifs des embryons immatures de Capsella bursa pastoris au cours de leur culture «in vitro». Actes du 101" congrès. Nat. Soc. Sav. Lille Sci. Fasc. 1, 595-606.

Murashige T., Skoog F., 1962. A revised medium for rapid growth and bioassays with tissue cultures. Physiol. Plantarum, 15, 473-497. 
Raghavan V., Torrey J.G., 1964. Effects of certains growth substances on the growth and morphogenesis of immature embryos of Capsella in culture. Plant. Physiol., 39, 691-699.

Rietsema J., Satina S., Blakeslee A.F., 1953. The effect of sucrose on the growth of Datura stramonium embryos «in vitro ». Am. J. Bot., 40, 538-545.

Rijven A.H.G.C., 1952. In vitro studies on the embryo of Capsella bursa pastoris. Acta. Bot. Neerl., 1, 157-200.

SimpSON, 1965. Dormancy studies in seed of Avena fatua. The role of gibbcrellin in embryo dormancy. Can. J. Bot., 43, 793-816.

Stone E.C., Duffield J.W., 1950. Hybrids of sugar Pine by embryo culture. J. For., 48, 200-201.

Тномлs M.J., 1970. Premières recherches sur les besoins nutritifs des embryons isolés du Pinus sylvestris L. Embryons différenciés. C.R. Acad. Sci., 270, 2648-2651.

Thomas M.J., 1980. Applications physiologiques de la culture d'embryons isolés. In : Connaissance de la biologie des arbres par les cultures in vitro. $2^{\circ}$ réunion de la Section Française de l'IAPTC sur l'arbre in vitro. Paris, 14-15 nov. 1980.

TuCKey H.B., 1933. Artificial culture of sweet cherry cmbryos. J. Hered., 24, 7-12.

Tuckey H.B., 1934. - Artificial culture methods for isolated embryos of deciduous fruits. Am. Soc. Hort. Sci. Proc., 32, 313.

Vieitez M., Vieitez E., 1980 a. Plantlet formation from embryonic tissue of chestnut grown in vitro. Plant. Physiol., 50, 127-130.

Vieitez M., Vieitez E., 1980 b. Culture of chestnut shoots buds in vitro. J. Hort. Sci., 55, 83-84. 